\title{
Article \\ Calculations of the Thermodynamic Characteristics and Physicochemical Properties of Symmetric and Asymmetric Isomeric Compounds for Identification in Chromatography-Mass Spectrometry
}

\author{
Elizaveta Fedorova ${ }^{1, *}$, Andrey Stavrianidi ${ }^{1,2}$, Irina Minenkova ${ }^{1}$ and Aleksey Buryak ${ }^{1}$ \\ 1 A.N. Frumkin Institute of Physical Chemistry and Electrochemistry Russian Academy of Sciences, \\ 31 Leninsky Prospect, GSP-1, 119071 Moscow, Russia; stavrianidi.andrey@analyt.chem.msu.ru (A.S.); \\ irina.vl.minenkova@mail.ru (I.M.); dir@phyche.ac.ru (A.B.) \\ 2 Chemistry Department, Lomonosov Moscow State University, 119991 Moscow, Russia \\ * Correspondence: elizaveta.chemi@gmail.com
}

Citation: Fedorova, E.; Stavrianidi, A.; Minenkova, I.; Buryak, A. Calculations of the Thermodynamic Characteristics and Physicochemical Properties of Symmetric and

Asymmetric Isomeric Compounds for Identification in ChromatographyMass Spectrometry. Symmetry 2021, 13, 1681. https://doi.org/10.3390/ sym13091681

Academic Editor: José Carlos R. Alcantud

Received: 3 August 2021

Accepted: 7 September 2021

Published: 12 September 2021

Publisher's Note: MDPI stays neutral with regard to jurisdictional claims in published maps and institutional affiliations.

Copyright: (c) 2021 by the authors. Licensee MDPI, Basel, Switzerland. This article is an open access article distributed under the terms and conditions of the Creative Commons Attribution (CC BY) license (https:// creativecommons.org/licenses/by/ $4.0 /)$.
Abstract: A combination of theoretical and experimental approaches was applied to determine the chromatographic rules of isomeric compounds' behavior for preliminary identification. In gas chromatography-mass spectrometry (GC-MS), identification is performed by spectra matching, however, difficulties arise with isomeric compounds, which cannot be distinguished from each other without additional information. The thermodynamic characteristics of the adsorption of symmetric and asymmetric isomers of chlorophenylphenols, dimethoxybiphenyls, tri- and tetrachlorobiphenyls were determined using molecular statistical calculations. By-products in the chlorination of 4hydroxybiphenyl were identified: 4-hydroxy-2,3' - and 3,2'-dichlorobiphenyls, 4-hydroxy-3,5,2' - and 2,3,6-trichlorobiphenyls. A developed theoretical approach was applied to predict the retention order of tri- and tetra-chlorobiphenyls. The GC-MS data and molecular statistical calculations made it possible to determine the main products of methoxybenzene dimerization as well as identify impurities. Thermodynamic parameters were received to describe the unusual retention behavior of epimers in reversed-phase high-performance liquid chromatography. Molecular descriptors were calculated to determine correlation with retention of both structural isomers and epimers. Descriptor combining surface area and partial charge information turned out to be useful in evaluating retention order for isomers.

Keywords: isomers; chromatography-mass spectrometry; thermodynamic characteristics

\section{Introduction}

Currently, chromatography coupled with mass spectrometry is one of the leading methods in qualitative and quantitative analysis of mixtures of various nature and composition. A special research direction in chromatography is the study of mixtures containing isomeric compounds. The geometric structure of molecules determines their physicochemical characteristics and, accordingly, chromatographic sorption behavior. The greatest differences in chromatographic behavior with differences in geometric structure appear for isomers. The relevance of research related to isomeric compounds is still of great importance due to the vastness of forms of isomerism, which can be transformed into one another. All forms of isomerism are usually divided into two classes: structural and stereoisomerism. Structural isomers include compounds in which different modes of binding in the molecule are observed, while stereoisomers are compounds with different spatial arrangements of atoms. Optical and geometric isomerism is distinguished when spatial isomers are considered [1,2]. Molecules of optical isomers lack a plane of symmetry. Geometric isomerism reflects the different positions of the substituents relative to the double bond or ring. A special kind of geometric isomerism is syn-anti-isomerism. This type of isomerism 
is similar to cis-trans-isomerism, while rotation around $\mathrm{C}=\mathrm{N}$ or $\mathrm{N}=\mathrm{N}$ bonds is observed (cis corresponds to syn, and trans to anti) [1]. Rotation of an atom or groups of atoms around a carbon-carbon simple bond (or other bonds) leads to the formation of conformational (rotational) isomers. The resulting isomers are called conformers, rotamers [3]. In gas chromatography (GC) the most common is gas-liquid chromatography that does not allow the separation of isomers based on differences in structure. The differences in the structure of the isomers are leveled out when dissolved in the stationary liquid phase. This may be due to the interaction of atoms of the adsorbed molecules with atoms of the liquid stationary phase.

Another type of GC is gas-adsorption chromatography, although rare. Non-porous materials such as graphite, boron nitride, and diamond are often used as sorbents in gas-adsorption chromatography. In this case, the plane of the adsorbent surface allows revealing the differences of molecules with lower and higher group dimensions. No change in the chromatographic adsorption is observed in case the atoms responsible for the differences in symmetry are located in the same plane. If the atoms causing the asymmetry of the isomers are in the plane perpendicular to the adsorption plane, then such molecules adsorb more weakly compared to more symmetric ones. Thus, the more symmetric the molecule is and the closer its atoms are to the surface, the higher the adsorption capacity and, accordingly, the chromatographic retention. Liquid adsorption chromatography allows distinguishing isomers on hypercarb or silica gel sorbents. However, it is necessary to take into account the influence of the mobile phase, which can hinder the sorption of molecules due to competitive adsorption. In the case of liquid adsorption chromatography, as in gas-liquid chromatography, dissolution occurs in the volume of the liquid phase. Molecules penetrate the volume of the grafted layer due to their specific orientation.

Separation systems in liquid chromatography are much more complicated due to eluent participation in the process of sorption of analytes on a sorbent surface. It is assumed that in reversed-phase high-performance liquid chromatography (RP-HPLC), both partition and adsorption govern the separation process [4]. Mass spectrometry allows the detection of unresolved peaks and provides structural information needed to annotate the compounds. The fragmentation pathways in tandem mass spectrometry (MS/MS) spectra could be different for structural isomers, while these spectra may be similar or identical for stereoisomers or some of the positional isomers. In this regard, the separation of such isomers is important for their identification. Great attention is placed on the separation of enantiomeric compounds having pharmaceutical applications [5,6]. However, separation and identification of positional isomers and epimers having potential bioactive properties could be not less complicated. Separation of some positional isomers on chiral stationary phases is well-studied, for instance, retention of ortho-, meta-, para xylenes on cyclodextrines is explained by spatial structure of the molecules. The linear structured para-xylene is fully included in the cyclodextrines, and as a result, has a larger retention time than its isomers. However, separation of positional isomers on the RP-HPLC cannot be unequivocally estimated. Moreover, the study of retention of different diastereoisomers and close positional isomers in RP-HPLC mass spectrometry (RP-HPLC-MS) conditions may shed light on the adsorption and partition mechanisms of their interaction with the stationary phase, which may lead to the correct prediction of their elution order.

Glycosides provide great scope for studying the retention and separation of structural isomers and diastereomers in reversed-phase. Glycosides are firmed by nonpolar aglycone and a polar part-a sugar side chain and their retention mechanism is highly configuration dependent. It has been shown that separation of epimeric steroidal glycosides from Tribulus terrestris L. cell culture can be achieved on a C18-bonded phase [7]. In addition, epimerization and dehydroxylation can take place during thermal treatment of plant material [8,9] or by further digestion in the living organisms [10-12]. Prominent antioxidation, anti-inflammation, and anti-aging activities of such triterpene glycosidecontaining preparations encourage the researchers to apply HPLC-MS analysis for their stereoselective determination and pharmacokinetic study $[12,13]$. Thus, it is important 
to perform correct annotation of the isomers. For this purpose, molecular properties and physical-chemical parameters calculated as a number of descriptors [14] may be used to find a correlation with retention. Thus, it remains to solve the regression problem using machine learning methods for prediction retention time or the order.

More traditional approaches of the prediction the main chromatographic, mass spectrometric, thermodynamic characteristics, and selection of the optimal conditions for the desired process are based on molecular dynamics, molecular-statistical, and quantummechanical calculations. The possibility and success of using the molecular statistical method for calculating the thermodynamic characteristics of adsorption on graphitized thermal carbon black (GTCB) were presented in [15]. Graphite thermal carbon black is a non-polar adsorbent with high geometric and chemical surface uniformity. Under the conditions of GC, selective separation of structural isomers is observed depending on their symmetry [16]. For flat surface adsorbents (e.g., GTCB), trans-isomers have an energetic advantage. The strongest retention is observed for the cis-isomers in the case of the distribution of the compound over the bulk phase.

The theoretical study of isomeric compounds are of particular high importance when the objects of research are health and environmentally hazardous. Since work with toxic substances requires special additional conditions, theoretical study of such substances seems to be more than promising. The purpose of this work was to improve the reliability of the identification of isomers of various natures and to establish the conditions for the analysis of complex industrial mixtures and environmental objects. To achieve this goal, a combination method was applied based on the data of GC-MS analysis and the results of molecular statistical calculations. The separation of epimers and structural isomers on the example of several triterpene and steroidal glycosides was investigated to enhance the understanding of the retention behavior of the complex naturally occurring molecules in RP-HPLC conditions.

\section{Materials and Methods}

Analytes preparation for GC-MS analysis. For thermal desorption, model samples were prepared in the form of powders of the corresponding materials with a grain size of $0.125-0.15 \mathrm{~mm}$ in order to increase their surface and make them easier to place in a pyrolyzer. The adsorbates were applied from solvent solutions. After holding, the samples were filtered off, dried in a flow of helium, and used for research. Deuterated or chlorinecontaining aromatic hydrocarbons in a highly volatile solvent were used as an internal standard. The weighed amount was $1-4 \mathrm{mg}$.

Analytes preparation for LC analysis. The following reagents were used in the work: ginsenosides Rg3, Rh1 (>98\%, Phytolab GmbH, Vestenbergsgreuth, Germany); ginsenosides Rh4, Rk3, Rk1, Rg5 (>98\%, ChromaDex Int., Los Angeles, CA, USA); acetonitrile (for gradient chromatography Panreac, Barcelona, Spain), acetic and formic acids (SigmaAldrich, Burlington, VT, USA). Deionized water was prepared from distilled water on a Milli-Q installation (Millipore, Burlington, VT, USA). An accurately weighed $1 \mathrm{mg}$ of each standard was dissolved in $1 \mathrm{~mL}$ of methanol. The resulting solutions were used to prepare a stock solution with ginsenoside concentrations at the level of $40 \mu \mathrm{g} / \mathrm{mL}$, which was stored at $-17^{\circ} \mathrm{C}$. The resulting stock solution was used to prepare the solution with a concentration equal to $1.25 \mu \mathrm{g} / \mathrm{mL}$ in $50 / 50 \%$ water/acetonitrile solvents. Retention data for 20(S) Rg3, 20(R) Rg3, 20(R) Rh1, 20(S) Rh1, and 20(S) Rg2, 20(R) Rg2 analyzed with RP-HPLC were obtained from the literature data [13].

To a sample of $3 \mathrm{mg}$ of the purified amount of steroidal glycosides of the biomass of Dioscorea deltoidea cell culture, provided for research by the staff of the Department of Plant Physiology, Faculty of Biology, Moscow State University, $1.5 \mathrm{~mL}$ of a solvent mixture of acetonitrile and water (50/50 vol.\%) was added. Then the mixture was kept at a temperature of no more than $30^{\circ} \mathrm{C}$ in an ultrasonic bath for $40 \mathrm{~min}$, stirring every $10 \mathrm{~min}$. After that, $1.5 \mathrm{~mL}$ of the extract was taken and passed through a $0.45 \mu \mathrm{m}$ Chromafil Xtra 
PET-45/25 syringe filter (Macherey-Nagel GmbH, Düren, Germany). Then the samples were diluted 10 times in a $10 \%$ aqueous solution of acetonitrile.

LC-MS conditions. HPLC-MS analysis was performed with MS/MS system consisted of a QTrap 3200 tandem mass spectrometer (AB Sciex, Concord, ON, Canada) equipped with electrospray ionization (ESI), and HPLC system ULTIMATE 3000 (Thermo Fisher Scientific, Waltham, MA, USA). Columns with a reversed-phase stationary phase Acclaim RSLC 120 C18 $(150 \times 3.0 \mathrm{~mm} ; 3.0 \mu \mathrm{m})$ and Acclaim C18 $(150 \times 3 \mathrm{~mm}, 3 \mu \mathrm{m})$, from Thermo Scientific (USA) were used in the determination of triterpene and steroidal glycosides respectively. The determination was carried out using an ESI source in scan mode in $m / z$ range 100-1350. The composition of the mobile phase for the steroidal saponins analysis: an aqueous solution of acetic acid $0.3 \%$ (eluent A) and acetonitrile (eluent B). The separation of the sample was carried out in an isocratic mode of supplying an eluent in a phase A/phase B ratio of $26.5 / 73.5$ (vol.\%); The flow rate was $0.25 \mathrm{~mL} / \mathrm{min}$. The temperature of the column thermostat was varied from $22-35^{\circ} \mathrm{C}$. The volume of the injected sample was $5 \mu \mathrm{L}$. The composition of the mobile phase for the ginsenoside analysis: an aqueous solution of formic acid $0.5 \%$ (eluent A) and acetonitrile (eluent B). Sample separation was carried out in a gradient mode; the flow rate was $0.5 \mathrm{~mL} / \mathrm{min}$. The temperature of the column thermostat was $25^{\circ} \mathrm{C}$. The injection volume was $10 \mu \mathrm{L}$.

GC-MS conditions. Studies were carried out on a JMS-D300 GC-MS equipped with a JMA-2000 computer and an HP 5890 chromatograph. Thermal desorption studies were carried out using direct injection in the temperature range from 30 to $400{ }^{\circ} \mathrm{C}$, and the sample heating rate varied from 2 to $50{ }^{\circ} \mathrm{C} / \mathrm{min}$. Chromatograms were recorded in the full-scan EI positive ion mode ( 40 to $450 \mathrm{~m} / z$ ). The temperature of the ion source was $150{ }^{\circ} \mathrm{C}$, the energy of ionizing electrons was $70 \mathrm{eV}$, the accelerating voltage $-3 \mathrm{kV}$, the mass range- $40-400$. Mass spectra were additionally investigated at low energy $10-15 \mathrm{eV}$ and in the chemical ionization with reactant gases methane, ammonia, and isobutane.

The chromatographic study was carried out on columns with GTCB $(50 \times 0.1 \mathrm{~cm}$, $70 \times 0.1 \mathrm{~cm}, 70 \times 0.08 \mathrm{~cm}, 120 \times 0.1 \mathrm{~cm})$ and a capillary column $(30 \mathrm{~m} \times 0.53 \mathrm{~mm} \times 1.5 \mu \mathrm{m})$ with a weakly polar phase DB-5 (5\% phenylmethylsilicone). Helium was used as a carrier gas. Carrier gas flow rate was $5-20 \mathrm{~mL} / \mathrm{min}$ for microfabricated columns and $2 \mathrm{~mL} / \mathrm{min}$ for capillary columns. Chromatographic separation was carried out on an HP 5890 chromatograph coupled to a mass spectrometer using a jet separator; when working with a capillary column, the sample was injected with a discharge of 1:10. To optimize chromatographic separation, different temperature programming rates were used from 1 to $25^{\circ} \mathrm{C} / \mathrm{min}$ for GTCB columns and from 0.5 to $15^{\circ} \mathrm{C} / \mathrm{min}$ for a capillary column. Identification was carried out using a library search (NIST MS Search software), or based on the laws of fragmentation.

\section{Computation Details}

Either the relative retention times were determined relative to the components of the mixture, identified by MS, or special standard substances with known values of Henry's constant were added. Since most of the chromatograms were obtained in temperature programming mode, the retention temperature was used instead of the retention volumes, i.e., the temperature at which the maximum of the peak appears on the chromatogram. Calculation of Henry constants for adsorption was carried out according to the article [17]. The calculation was performed using PC GAMESS software for quantumchemical calculations. The potential in the form of Buckingham-Corner was selected for the calculation of atom-atom potentials of the nitrogen atom with carbon atoms of the basal face of graphite.

Stereo SMILES of each molecule were obtained with the OpenBabel GUI software (3.0.0). SMI files were further used for computing 3D coordinates and energy minimization with the command-line program "obabel". Energy minimization was performed in the MMFF94 force field with the steepest descent algorithm with 5500 steps. Molecules properties were calculated with R programming language with the "rcdk" (3.5.0) package. 


\section{Results and Discussion}

\subsection{Analyzing Isomers with GC-MS}

\subsubsection{Chlorophenylphenols}

The identification of macro and micro components in the chlorination of 4-phenylphenol under conditions simulating the operation of a water treatment plant was considered [18]. The total ion current (TIC) chromatogram of the mixture is presented in Figure 1a. It was found that the mixture contains one mono-, three di- and two tri-chlorophenylphenols. According to the chromatogram in Figure 1a, the most intensive peak determined by the library search corresponded to 4-hydroxy-3,5-dichlorobiphenyl. The mass spectrum of the main component of the mixture is shown in Figure $1 \mathrm{~b}$.
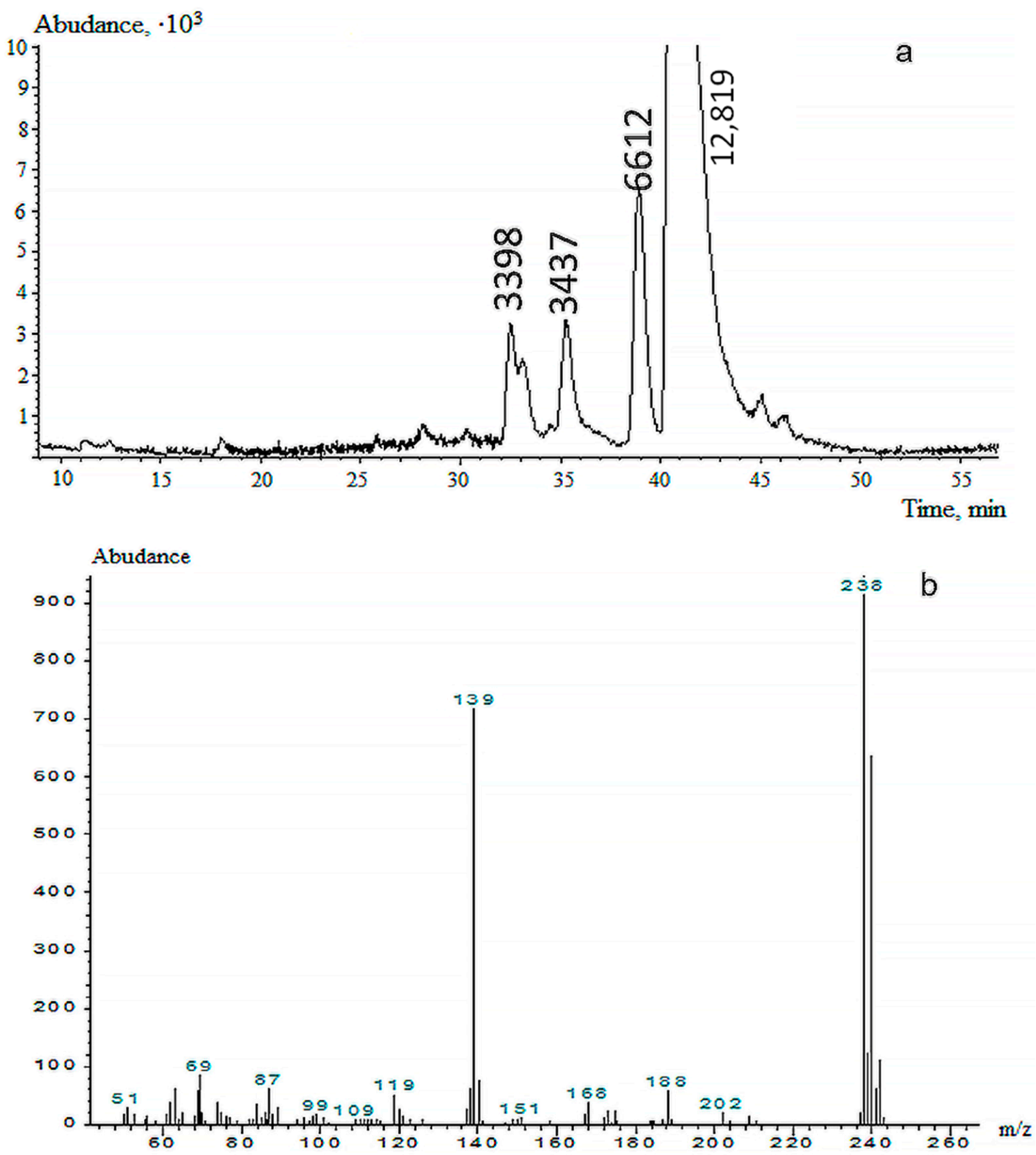

Figure 1. (a) TIC for a mixture of mono-, di- and trichloro- 4-hydroxybiphenyl, obtained on a column with GTCB and (b) the mass spectrum of the maximum component of the mixture, identified using the library search program as 4-hydroxy-3,5-dichlorobiphenyl. 
In order to evaluate the effective separation of isomers of the mixture, a chromatogram was obtained on a capillary column. Chromatograms were obtained for the main characteristic ions (Figure 2) based on the analysis of the chromatographic study of a mixture of isomers of the chlorination products of 4-hydroxybiphenyl. It should be noted that the total number of chromatographic peaks in the chromatograms obtained for different columns is the same. This fact indicates a high selectivity of graphitized carbon black for the studied mixture of isomers.
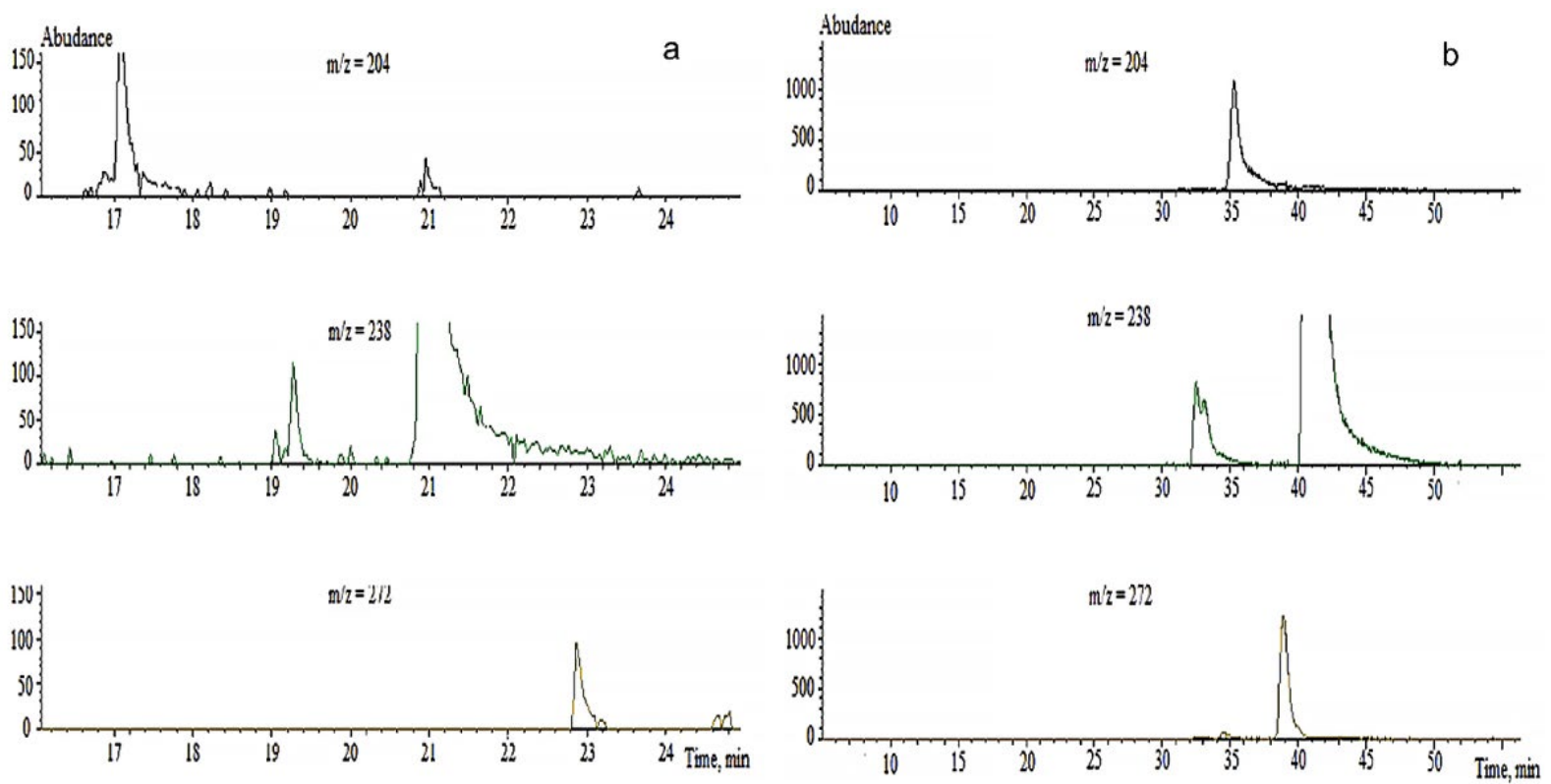

Figure 2. (a) Extracted ion chromatograms (EIC) for molecular ions of mono-, di- and trichloro- 4-hydroxybiphenyls $(m / z=204,238,272)$, obtained on a capillary column $(\mathbf{b})$ and a column with GTCB.

A library search in the database revealed the presence of 4-hydroxy-3-chlorobiphenyl and 4-hydroxy-3,5-dichlorobiphenyl in the mixture. However, the available data were insufficient to identify other compounds. Based on theoretical concepts of the chlorination process and the effect of substituents, it is impossible to predict with a high probability the full composition of the isomeric mixture. It should be noted that chlorine and phenyl activate the benzene ring in different ways. Secondly, since the process is not fully understood, the databases lack sufficient sets of mass spectra for a full analysis of the mixture. It is known that the methylation of isomers leads to differences in mass spectra [19]. Mass spectra for isomeric compounds were identical and the randomization of substituents was observed. Figure 3 shows examples of mass spectra of isomeric dichlorobiphenyls.

The analysis of mass-spectrum in Figure 3 revealed that the fragmentation of these isomers was different from the fragmentation of 4-hydroxy-3,5-dichlorobiphenyl (Figure 1b). The main difference in mass-spectra may be caused by the arrangement of the substituents. The intensity of the fragment ion $(139 \mathrm{~m} / z)$ is significantly higher than for the molecular ion $(238 \mathrm{~m} / \mathrm{z})$ for 4-hydroxy-3,5-dichlorobiphenyl. (Figure 1b). Due to the lack of sufficient data to identify the remaining components of the mixture, molecular statistical calculations of chromatographic characteristics were performed.

Molecular statistical calculations were performed with interatomic potential for the oxygen atom in the hydroxyl group. In this case, the influence of internal rotation on adsorption was taken into account. The results obtained for isomers with different contents of chlorine atoms in the ortho-position are summarized in Table 1. For comparison, Table 1 shows the data for 4-hydroxy-3,5, $4^{\prime}$-trichlorobiphenyl, which does not contain substituents in the ortho-position. 

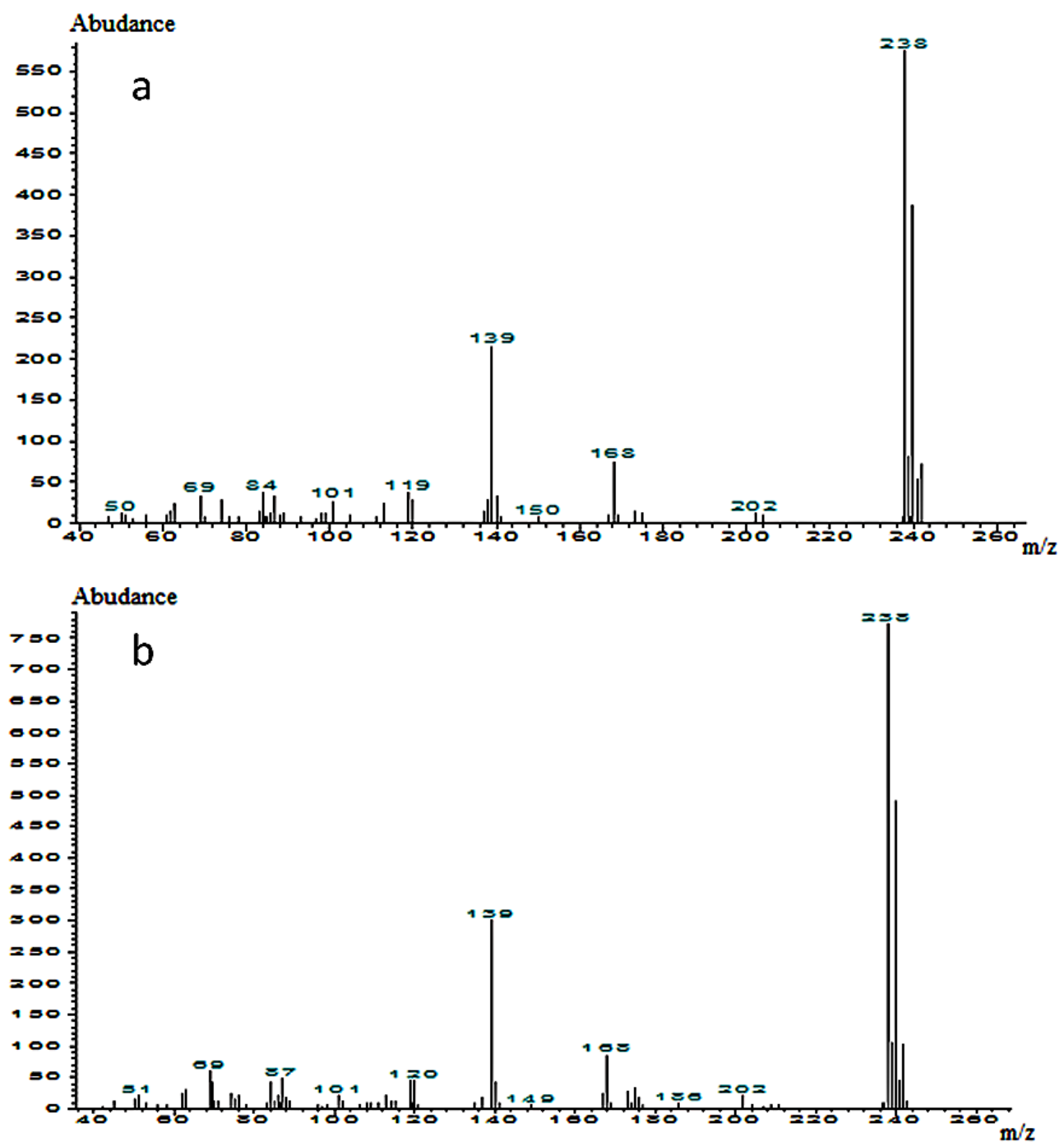

Figure 3. Mass spectra of isomeric (a) 4-hydroxy-2, $3^{\prime}$ - dichlorobiphenyl and (b). 4-hydroxy-3,2' dichlorobiphenyl.

Table 1. Henry constants of chlorophenylphenols (at $550 \mathrm{~K}$ ) and the observed elution order in the column with GTCB.

\begin{tabular}{|c|c|c|c|c|c|c|}
\hline \multirow{2}{*}{$\begin{array}{l}\text { Position of } \\
\text { Substitutes * }\end{array}$} & \multirow[b]{2}{*}{ Inner Corner } & \multicolumn{3}{|c|}{ Henrys' Constants } & \multicolumn{2}{|c|}{ Elution Order } \\
\hline & & $\begin{array}{c}\text { One } \\
\text { Substitute }\end{array}$ & $\begin{array}{c}\text { Two } \\
\text { Substitutes }\end{array}$ & $\begin{array}{c}\text { Three } \\
\text { Substitutes }\end{array}$ & Supposed & Calculated \\
\hline $3,2^{\prime}-(A)$ & 53 & & 6.52 & & $1,2(?)$ & 1 \\
\hline $2,3^{\prime}-(S)$ & 53 & & 6.58 & & 1,2 (?) & 2 \\
\hline $3,5,2^{\prime}-$ & 53 & & & 6.65 & $3(?)$ & 3 \\
\hline $3,2^{\prime}, 4^{\prime}-(A)$ & 53 & & & 6.75 & $3(?)$ & \\
\hline $2,5,2^{\prime}-(S)$ & 65 & & & 6.76 & $3(?)$ & \\
\hline $3-$ & 38 & 7.56 & & & 4 & 4 \\
\hline $3,2^{\prime}, 4^{\prime}-(S)$ & 53 & & & 7.96 & $5(?)$ & \\
\hline $2,3,6-$ & 64 & & & 8.22 & $5(?)$ & 5 \\
\hline $3,5-$ & 38 & & 8.35 & & 6 & 6 \\
\hline $3,5,4^{\prime}-$ & 38 & & & 10.09 & & \\
\hline
\end{tabular}

* The indices $S$ and $A$ indicate that the calculations were carried out for the "syn" or "anti" conformations, respectively. 
According to the calculated data, the retention of 4-hydroxy-3,5-dichlorobiphenyl is significantly lower than for 4-hydroxy-3,5,4'- trichlorobiphenyl. It is possible to select compounds corresponding to the observed order of isomer elution and degree of substitution taking into account the relative position of isomers in the chromatogram (Figure 2b). At the same time, due to a large number of isomers, the identification of compounds cannot be carried out correctly enough. However, it can be affirmed that the dichlorophenylphenols in the mixture are 4-hydroxy-2,3'- and 3,2'-dichlorobiphenyls, and trichlorophenylphenols are 4-hydroxy-3,5,2' - and 2,3,6-trichlorobiphenyls taking into account all available data.

\subsubsection{Dimethoxybiphenyls}

Dimethoxy-substituted biphenyls are also of concern. During the catalytic dimerization of methoxybenzene such by-products as isomers of dimethoxybiphenyl are formed. The content of these by-products in the mixture can be comparable with the amount of the main product [20]. It is impossible to predict the class of formed by-products based only on the catalytic laws of this type of reaction.

The results of the GC-MS study of the isomeric mixture of dimethoxy-substituted biphenyl are shown in Figure 4. It was found that in the mixture, in addition to two isomers of dimethoxy-substituted biphenyl, three isomers of the by-product are also present. It can be assumed that the isomeric impurities are derivatives of biphenyl, diphenylmethane, or dibenzofuran based on mass-spectrometric studies.
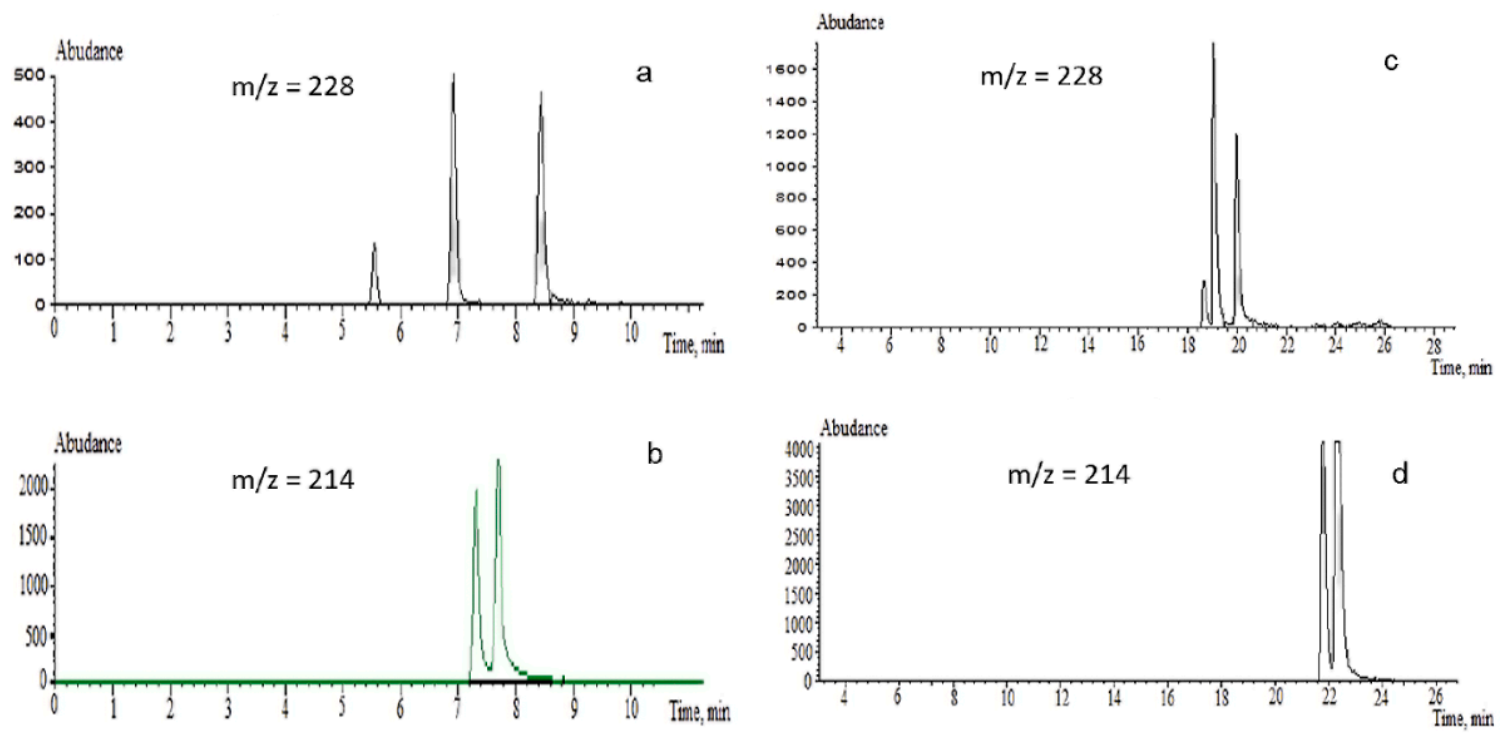

Figure 4. EIC obtained on capillary $(\mathbf{a}, \mathbf{b})$ and GTCB $(\mathbf{c}, \mathbf{d})$ columns, based on characteristic ions of isomeric dimethoxydiphenyls $(\mathbf{b}, \mathbf{d})$ and impurities $(\mathbf{a}, \mathbf{c})$.

The parameters of the geometric structure of the molecules were taken from studies on electron diffraction [21] or were determined as the average of the angles and bond lengths of molecules of the same compound class to carry out molecular statistical calculations. It should be noted that the studied derivatives are characterized by internal rotation relative to the bond connecting the benzene rings. The rotation of the methoxy group relative to the oxygen-carbon bond of the benzene ring ( $\mathrm{C}-\mathrm{OMe}$ ) is also possible. In this case, the rotation of the methyl group relative to the carbon-oxygen bond (O-CH3) can be neglected, since it has no effect on adsorption and on the chromatographic behavior of molecules. This assumption was made on the basis of calculations of the thermodynamic characteristics of adsorption and barriers to internal rotation in methyl derivatives of benzene [22].

Twelve isomers can be formed, six of which contain -OCH3 groups in different positions for dimethoxybiphenyls. The calculations for such isomers were carried out since in methoxybenzene dimerization no migration of the methoxy group is observed, and the 
following isomers are formed: $2,2^{\prime}-, 2,3^{\prime}-, 2,4^{\prime}-, 3,3^{\prime}-, 3,4^{\prime}$ - and 4, $4^{\prime}$ - dimethoxybiphenyls [23]. Only $2,2^{\prime}$ - dimethoxybiphenyl has a characteristic mass spectrum and can be identified by GC-MS. Based on the fact that the -OCH3 group in the ortho-position (with respect to the bond between the benzene rings) can increase the angle of internal rotation of isomeric dimethoxybiphenyls, while reducing steric hindrance, it can be assumed that the methoxy group is close to the methyl. In order to study the effect of internal rotation on TCA (thermodynamic characteristics of adsorption), the angles of internal rotation of methoxy groups for 2,2' - dimethoxybiphenyl were varied, keeping the angle of internal rotation of benzene rings in the "syn" conformation equal to $70^{\circ}$. Figure 5 illustrates the obtained results.

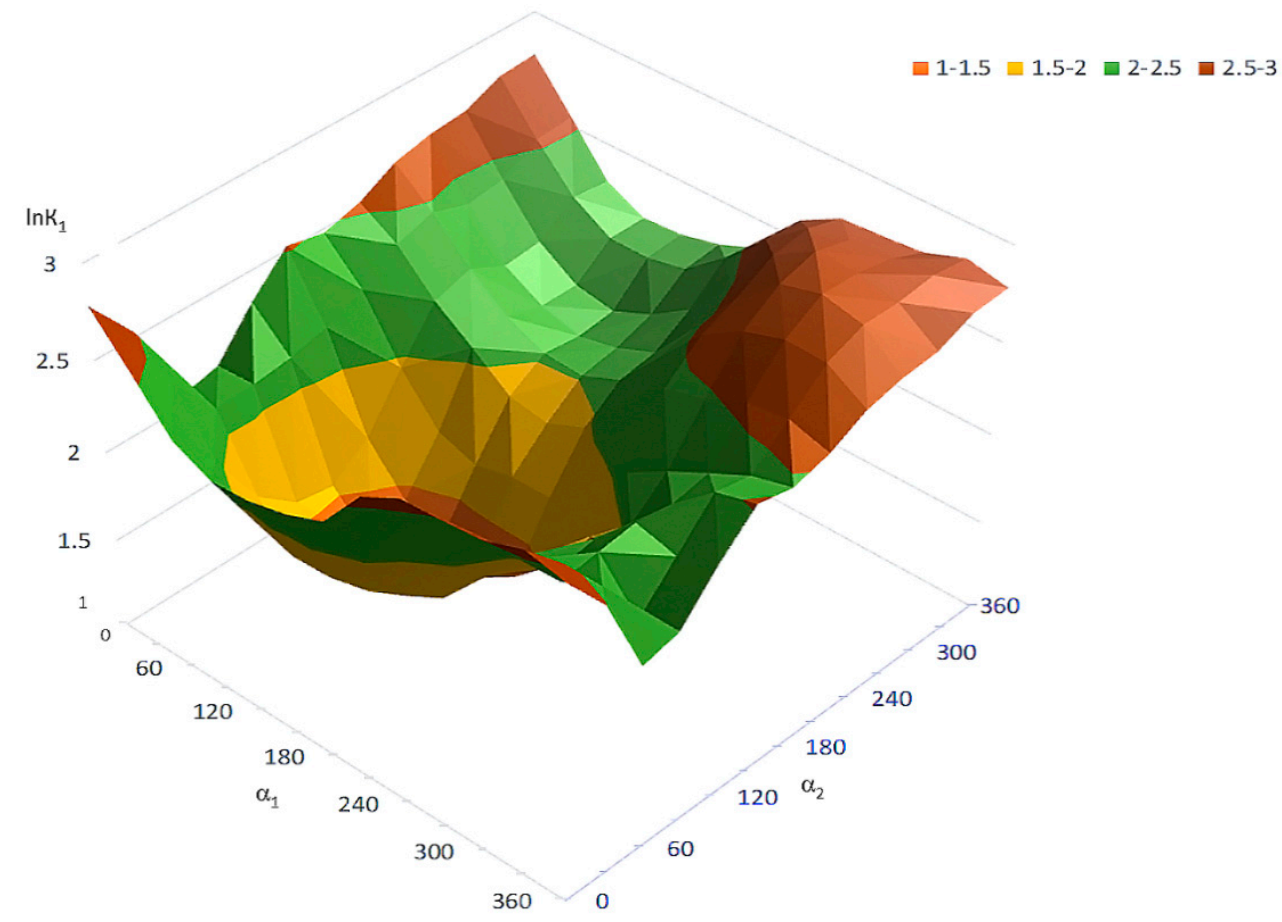

Figure 5. Dependence of $\operatorname{lnK}$ on the angles of internal rotation of methoxy groups for 2,2'-dimethoxybiphenyl at $525 \mathrm{~K}$.

According to Figure 5, the rotation of the - $\mathrm{OCH} 3$ groups changes the $\ln \mathrm{K} 1$ values from 1 to 3 , similar changes were observed with the rotation of the inner angle in biphenyl. Thus, in further calculations the angle of internal rotation for isomers $2,3^{\prime}$ - and $2,4^{\prime}$ - dimethoxybiphenyls was taken equal to $54^{\circ}$ [22]. The logarithm values of Henry's constants depending on the angle of rotation of the methoxy group are given in Table 2.

Table 2. Values of $\operatorname{lnK} 1$ at $500 \mathrm{~K}$ for 2,3'- and 2,4'-dimethoxybenzenes at various angles of internal rotation of methoxy groups.

\begin{tabular}{ccccc}
\hline \multirow{2}{*}{ Substance } & \multicolumn{4}{c}{ Internal Rotation Angle of Methoxy Groups $\left(\boldsymbol{\alpha}_{\mathbf{1}}\right)$} \\
\cline { 2 - 5 } & $\mathbf{4 0}$ & $\mathbf{4 5}$ & $\mathbf{5 0}$ & $\mathbf{5 5}$ \\
\hline 2,3'-dimethoxybenzene & 7.50 & 6.40 & 5.20 & 4.45 \\
\hline 2,4'-dimethoxybenzene & 7.40 & 6.20 & 5.00 & 4.86 \\
\hline
\end{tabular}

The retention characteristics for isomers with substituents in the ortho-position are lower than for isomers with substituents in other positions with respect to the bond between benzene rings (Table 2). Consequently, ortho-substituted dimethoxybiphenyls were not reaction products of the methoxybenzene catalytic dimerization. The retention characteristics for compounds without ortho-substituents differ less (Table 3). The main products of 
methoxybenzene dimerization in the test mixture were $3,4^{\prime}$ - and $4,4^{\prime}$ - dimethoxybiphenyls (Table 3).

Table 3. $\ln K 1$ values for isomeric dimethoxydiphenyls at different temperatures.

\begin{tabular}{ccccc}
\hline \multirow{2}{*}{ Substance } & \multicolumn{3}{c}{ T, K } \\
\cline { 2 - 5 } & $\mathbf{4 0 0}$ & $\mathbf{4 5 0}$ & $\mathbf{5 0 0}$ & $\mathbf{5 5 0}$ \\
\hline 2,2'-dimethoxybiphenyl & 6.23 & 4.06 & 2.36 & 1.01 \\
2,3'-dimethoxybiphenyl & 8.78 & 6.33 & 4.39 & 2.83 \\
2,4'-dimethoxybiphenyl & 9.36 & 6.78 & 4.76 & 3.12 \\
3,3'-dimethoxybiphenyl & 10.71 & 7.89 & 5.67 & 3.89 \\
3,4'-dimethoxybiphenyl & 11.51 & 8.66 & 6.40 & 4.57 \\
4,4'-dimethoxybiphenyl & 12.40 & 9.45 & 7.16 & 5.21 \\
\hline
\end{tabular}

The next stage of the study was the identification of impurities from the catalytic dimerization of methoxybenzene. Impurities were found to be isomers based on the mass spectra (Figure 6).
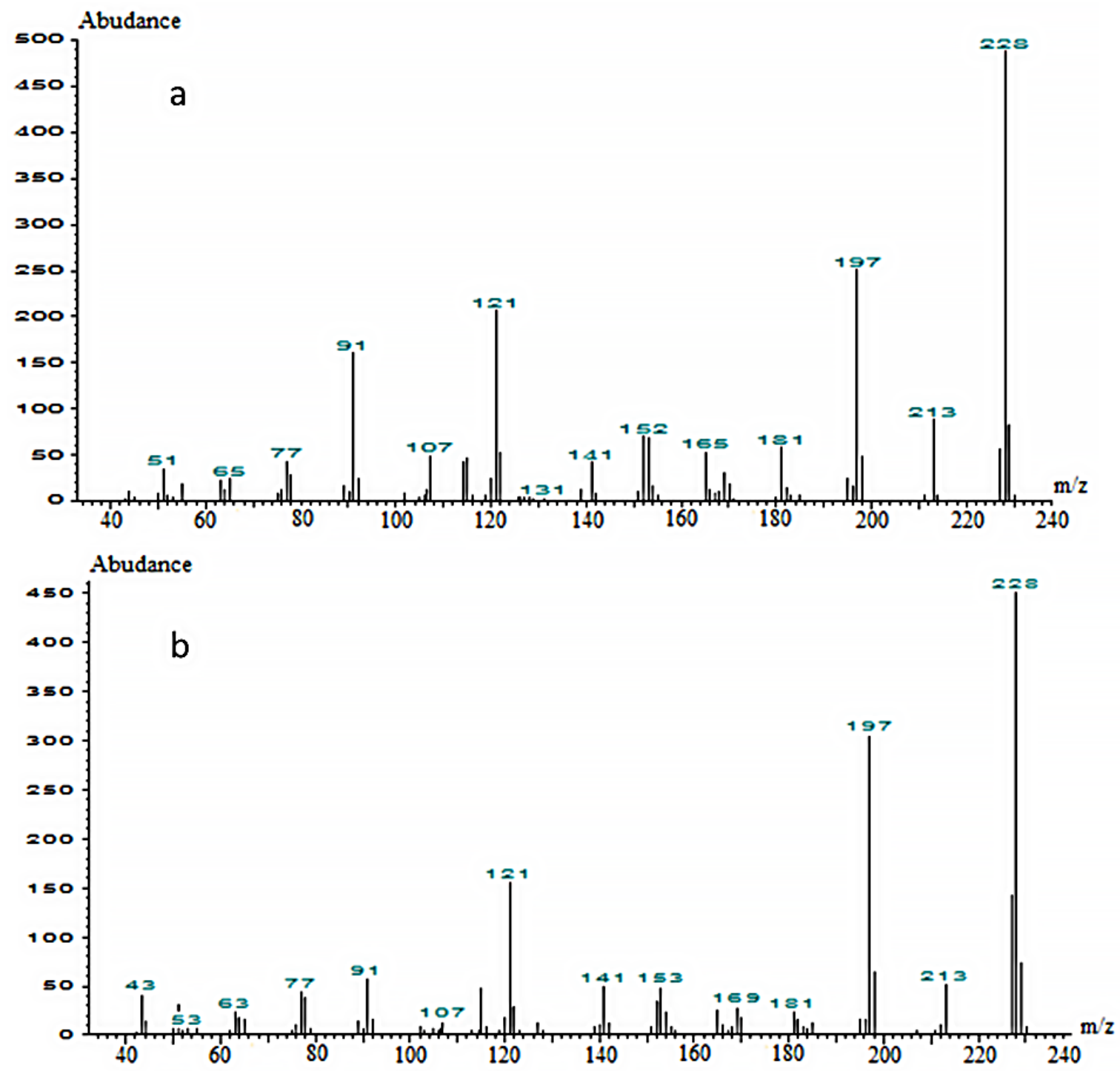

Figure 6. Mass spectra of impurities formed during the dimerization of methoxybenzene (a) 2,6-dimethoxybiphenyl, (b) 2,2'- dimethoxybiphenyl. 
We can assume several possible structures based on the theoretical prerequisites for the formation of by-products [24] and the molecular weight of impurities. To reduce the number of possible candidates, a comparative analysis of TCA of suspected compounds was carried out. According to the study [25], methoxy-substituted biphenyls have different retention times on the column with GTCB, while the greatest differences in TCA were observed for the derivatives of diphenylmethane and dibenzofuran. Thus, we can conclude that the considered compounds belong to derivatives of 3- or 4-methyldiphenyl. It can be assumed that the impurities were 3 out of 36 possible isomers of methyloxydiphenyl. At the next step of the study, structures containing ortho-substituents for the bond between the benzene rings were selected. The following values of the angles of internal rotation were taken for molecular statistical calculations: for those containing only a methyl group- $54^{\circ}$, only a methoxy group $-54^{\circ}$, methyl and methoxy groups $-70^{\circ}$, two methoxy groups$70^{\circ}$, and three substituents $-77^{\circ}$. If the substituents were in the $2,2^{\prime}$-position, the syn conformation of the isomer was used for calculations.

According to the obtained results, 6-methyl-2,2'-dimethoxybiphenyl (MDMB) had the lowest retention, even in comparison with biphenyl. Ortho- unsubstituted MDMB should have the largest retention time because it more strongly retains than diphenyl and isomeric dimethoxybiphenyls. The fragmentation of compounds with a methyl group in the ortho-position in the mass spectrum is characterized by an increase in the intensity of the $[\mathrm{M}+\mathrm{H}]^{+}$ion, compared to ortho-unsubstituted compounds. This fact was used in the identification of isomers. The presence of a methoxy group in the ortho position also leads to an increase in the intensity of the peak of the [M-OCH3] ${ }^{+}$ion in the mass spectrum. These peaks in the mass spectra are specific to the impurities determined for the mixture. Fragmentation of compounds with substituents at 2,6- and 2,2'-positions should be different based on the study of chlorobiphenyls [26]. The retention times of isomeric impurities were between biphenyl and ortho-unsubstituted dimethoxybiphenyls. These compounds include MDMBs, which contain two substituents at the 2,6- and 2,2'-positions. Therefore, considered compounds should contain two methoxy groups in the ortho positions based on the obtained spectra and retention data, which could be 3-methyl-, 4-methyl-, and 5-methyl-2,2'-dimethoxydiphenyl. For these compounds, Henry's constants were calculated, which turned out to be: 4.91 for 3-methyl-, 5.09 for 5-methyl-, 5.21 for 4-methyl$2,2^{\prime}$-dimethoxydiphenyl. Thus, these compounds were isomeric impurities obtained in methoxybenzene dimerization reaction.

\subsubsection{Tri- and Tetrachlorobiphenyls}

Tri- and tetrachlorobiphenyls are either theoretically important. It is necessary to use a large number of isostructural fragments since polychlorinated biphenyls are complex molecules for TCA calculation. Biphenyl derivatives are characterized by the ability to rotate internally with respect to the bond connecting the benzene rings. Internal rotation reduces the interaction between the atoms in the ortho-position to the bond connecting the benzene rings, while it does not change the interaction of the ortho-substituents with the carbon atoms of the benzene rings located in the 1 and $1^{\prime}$ positions. This interaction is practically identical to the interaction of the carbon atom in position 8 of the naphthalene ring with the chlorine atom in position 1, for example, in 1-chloronaphthalene.

The difficulty of the TCA calculation for chlorobiphenyls and elution order prediction of the isomers from a column with a GTCB using traditional calculations is related to the lack of geometric parameters and refined interatomic potential. Two conformers differ in the angles of internal rotation can exist for asymmetric molecules, for example, 2,3,2'-trichlorobiphenyl. Since the geometric structure of molecules in the adsorbed state is unknown, the calculations were performed for the two possible conformations, syn and anti. The geometrical structure of other practically important isomeric chlorobiphenyls has been studied insufficiently. Many unsymmetrical chlorobiphenyls have several conformations, which makes TCA calculations especially difficult due to the fundamental uncertainty of the geometric structure. The main goal in calculating the TCA of isomeric chlorobiphenyls was 
to predict the correct elution order of isomers from the GTCB column. A comparison with the experimentally observed elution order for isomers was used to assess the correctness of the angles of internal rotation and interatomic potential parameters. Figure 7 shows a chromatogram of a mixture of 2,2'-, 2,6,2'-, 2,6,2', $6^{\prime}$-chlorobiphenyls and naphthalene as a standard. Mass chromatograms for the characteristic ions of these compounds suggest that $2,2^{\prime}$-substituted chlorobiphenyl has an intermediate retention and is located between tetra- and tri-substituted chlorobiphenyls. The TCA calculations were carried out taking into account the correction for the ortho effect for atoms located in the ortho position to the bond connecting the benzene rings, which was determined from the data on the adsorption of 1-chloronaphthalene.
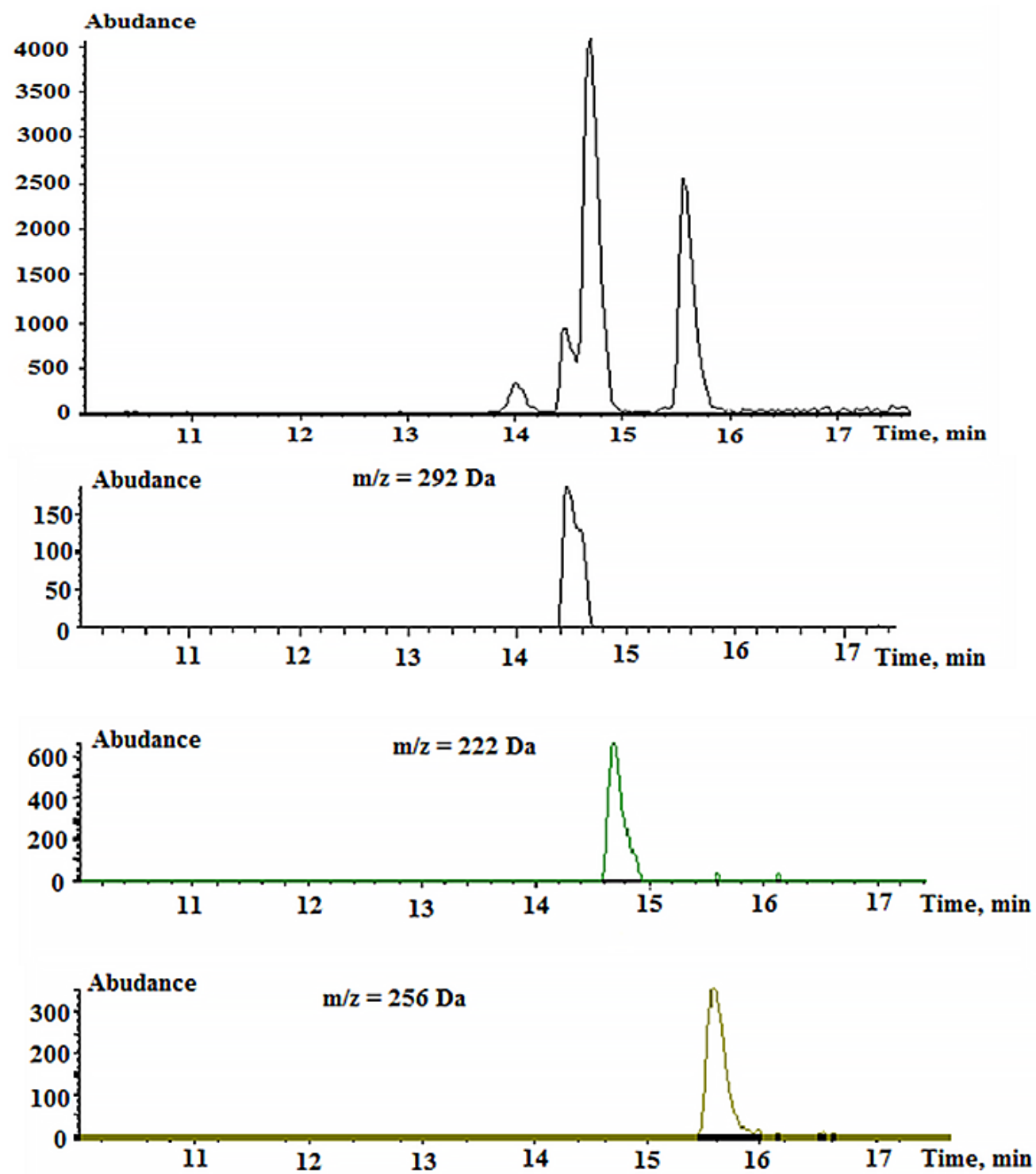

Figure 7. EIC of chlorobiphenyls.

The correct retention order for all compounds, taking into account the experimental values of Henrys' constants for naphthalene and 2,6,2'-trichlorobiphenyl, was obtained using angles of internal rotation equal to $60^{\circ}$ in the case of $2,2^{\prime}$, and $53^{\circ}$ in the case of $2,6,2^{\prime}, 6^{\prime}$-chlorobiphenyls. These effective values of the angles of internal rotation were used for further calculations of other isomers with the same isostructural fragment. In addition, the possible geometry distortion of the molecule with ortho-chlorine atoms out of the plane of the benzene ring was not taken into account. For 2,2'-substituted isomers, all calculations were performed for the syn-conformation. Agreement between the predicted 
and experimentally observed elution order of isomers was obtained based on the calculated retention values (Table 4 ).

Table 4. Values of the calculated and predicted elution order of some isomeric chlorobiphenyls from a column with a carbon sorbent.

\begin{tabular}{cccc}
\hline \multirow{2}{*}{$\begin{array}{c}\text { The Position of the Substituents in } \\
\text { the Chlorobiphenyl Molecule }\end{array}$} & lnK1, 500 K & \multicolumn{2}{c}{ Elution Order } \\
\cline { 3 - 4 } & & Experiment & Calculation \\
\hline $2,4,4^{\prime}$ & 5.4 & - & 2 \\
$2,4,6$ & 4.1 & 2 & 1 \\
$2,5,4^{\prime}$ & 4.9 & 3 & 2 \\
$3,4,4^{\prime}$ & 7.0 & - & 1 \\
$2,3,2^{\prime}, 6^{\prime}$ & 3.2 & 1 & 1 \\
$2,5,2^{\prime}, 6^{\prime}$ & 3.1 & 2 & 2 \\
$2,3,3^{\prime}, 4^{\prime}$ & 5.0 & $1-2$ & $1-2$ \\
$2,3,3^{\prime}, 5^{\prime}$ & 4.2 & - & 2 \\
$2,4,3^{\prime}, 5^{\prime}$ & 4.8 & - & $1-2$ \\
$2,6,3^{\prime}, 4^{\prime}$ & 4.2 & - & 1 \\
$2,6,3^{\prime}, 5^{\prime}$ & 3.7 & - & 3 \\
$3,4,3^{\prime}, 4^{\prime}$ & 7.0 & & \\
\hline
\end{tabular}

\subsection{Analyzing Isomers with RP-HPLC}

Chromatographic behavior in RP-HPLC of complex isomeric molecules on the example of saponins was studied. Two types of isomerism were considered: chain (position of multiple bonds) and diastereomers (epimers). The applicability of calculated properties for the description of the retention process and ability of prediction RT order was investigated. Chromatographic behavior for chain isomers was studied for triterpene ginsenosides. We considered two pairs of isomers: $\mathrm{Rk} 1, \mathrm{Rk} 3, \mathrm{Rh} 4$, and Rg5. The part of the chromatogram and their structures are presented in Figure 8.

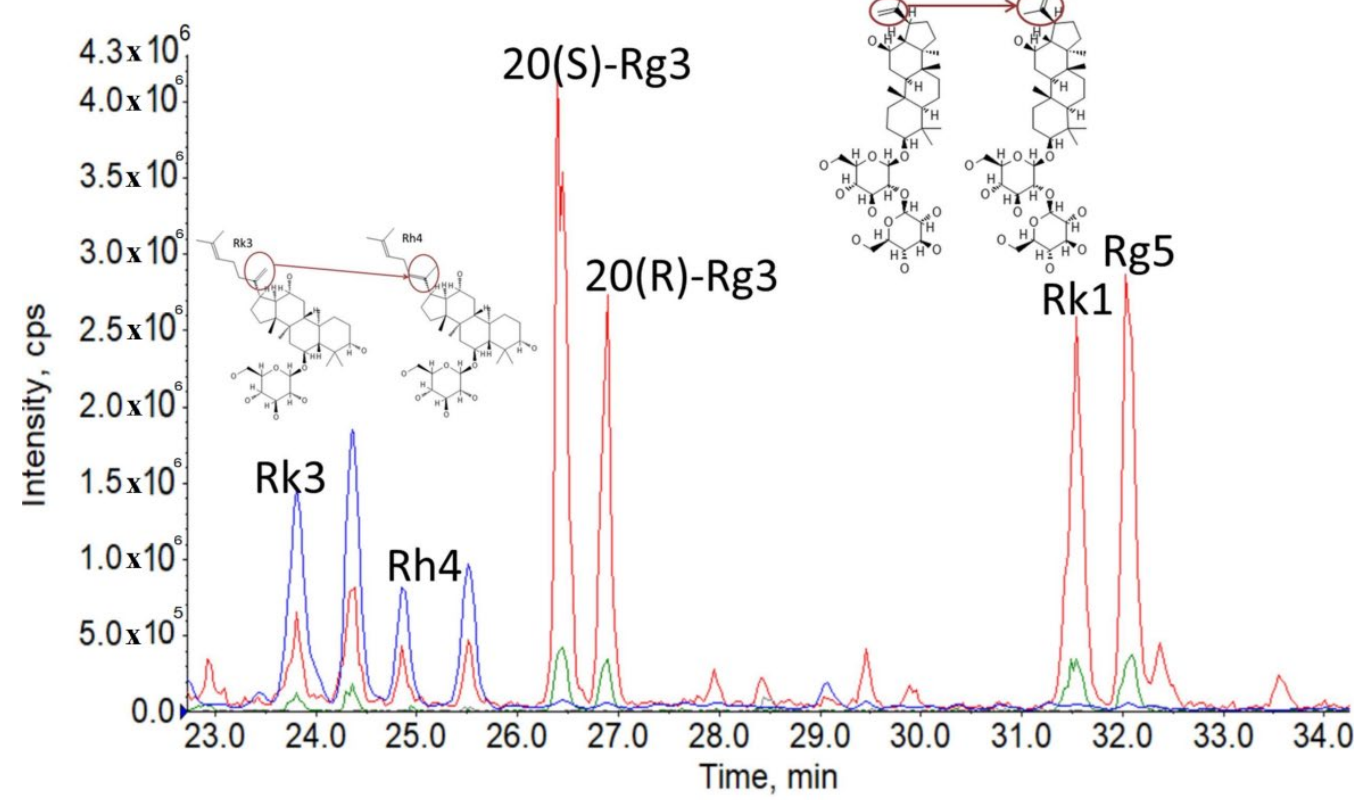

Figure 8. Selected ion chromatograms of chain isomers ( $R k 3$ and $R h 4, R k 1$ and $R g 5$ ) and epimers (20(S)-Rg3 and 20(S)-Rg3). Blue refers to ginsenosides with protopanaxatriol sapogenin, redprotopanaxadiol sapogenin, and green is for sapogenin derivative from oleanolic acid.

Ginsenosides Rk3 and Rh4 possess protopanaxatriol sapogenin, while Rk1 and Rg5 have protopanaxadiol sapogenin, which directly determines their chromatographic behav- 
ior. Rk1 and Rg5 ginsenosides elute later than $\mathrm{Rk} 3$ and $\mathrm{Rh} 4$ due to the absence presence of hydroxyl group at the C6 position in the aglycone, although having two sugars as substitutes. However, there is no obvious factor that can indicate the elution order of isomers with different multiple bond positions. Two calculated properties were used to describe the retention behavior of the chain isomers: the most common- $\log P$ and THSA - the sum of solvent accessible surface areas of atoms with an absolute value of partial charges less than 0.2 [27]. A measure of hydrophobicity $(\log P)$ used to predict chromatographic behavior in RP-HPLC was calculated based on the atom-type method in the absence of experimental data: MLogP, $\mathrm{X} \log \mathrm{P}, \mathrm{ALog} \mathrm{P}$, and $\mathrm{ALog} \mathrm{P} 2$. The simplest $\log \mathrm{P}$ is the MLogP whose prediction is based on the number of carbon and heteroatoms. MLogP correlates with RT, however, it does not distinguish molecules with one molecular formula but different structures. ALogP was more sensitive to the number of sugar substitutes and showed the inverse to RT values. $\mathrm{X} \log \mathrm{P}$ for a given compound is obtained by summing the contributions from component atoms and correction factors. Summarizing the results on $\log P$ calculations, $X \log P$ seems to be the most accurate in hydrophobicity calculation and appropriate to predict the elution order of chain isomers (Table 5).

Table 5. Calculated properties for chain isomers.

\begin{tabular}{ccccccc}
\hline Compound & Rt, $\min$ & XLogP & MLogP & ALogP & ALogp2 & THSA \\
\hline Rk3 & 23.87 & 6.03 & 4.54 & 3.89 & 15.16 & 650.38 \\
Rh4 & 24.48 & 6.17 & 4.54 & 3.84 & 14.74 & 654.28 \\
Rk1 & 31.6 & 6.36 & 4.76 & 3.32 & 11.00 & 750.38 \\
Rg5 & 32.15 & 6.51 & 4.76 & 3.26 & 10.64 & 776.80 \\
\hline
\end{tabular}

THSA was used as an alternative to $\log P$ property while both can characterize the retention behavior in RP-HPLC. THSA may be simply described as the total hydrophobic area of a molecule. The suggestion that more hydrophobic molecules should have a higher THSA value, which correlates with RT, was made. The supposed dependence was approved with calculated THSA values (Table 6). The increase of THSA was observed with increasing RT. XLogP and THSA had a linear correlation with RT for chain isomers and can be directly implemented in the identification of chain isomers in LC-MS analysis. Thus, THSA and XLogP were further calculated for epimers.

Table 6. Calculation results from Van't Hoff plots for steroidal saponins.

\begin{tabular}{cccccc}
\hline Compound Name & $\mathbf{R T}, \min \left(\mathbf{a t} \mathbf{3 5}{ }^{\circ} \mathbf{C}\right)$ & Equation & $\mathbf{R}^{2}$ & $\Delta \mathbf{H}, \mathbf{k J} / \mathbf{m o l}$ & $\Delta \mathbf{G}, \mathbf{k J} / \mathbf{m o l}$ \\
\hline 25(R)-protodioscin & 15.82 & $\mathrm{y}=-1328.7 x+6.0001$ & 0.99 & 11.1 & -4.2 \\
25(S)-protodioscin & 15.10 & $\mathrm{y}=-1367.1 \mathrm{x}+6.0693$ & 0.98 & 11.4 & -4.0 \\
25(R)-deltoside & 14.38 & $\mathrm{y}=-1236.5 \mathrm{x}+5.6211$ & 0.98 & 10.3 & -3.8 \\
25(S)-deltoside & 13.74 & $\mathrm{y}=-1290.2 \mathrm{x}+5.704$ & 0.97 & 10.7 & -3.7 \\
\hline
\end{tabular}

Chromatographic behavior of epimers was studied for triterpene and steroidal glycosides. Triterpene's elution order was the following: Rh1, Rg2, and the last was Rg3. Rh1 and $\mathrm{Rg} 2$ have protoponaxatriol sapogenin while $\mathrm{Rg} 3$ is diol, thus as well as for chain isomers, triols eluted earlier than Rg3. Thus, XLogP should accurately describe the hydrophobicity as for chain isomers. However, $\mathrm{X} \log \mathrm{P}$ for Rg2 was lower than for Rh1. This error can be due to extra sugar substitute (rhamnose) in the Rg2, which extensively contributed to the $X \operatorname{LogP}$ value. Despite the fact that epimers have slightly different chemical and physical properties, neither XLogP nor other calculated atom-based LogP is not cable to distinguish epimers. Thus, we have chosen THSA as the most sensitive to the complicated structure retention dependence factor. Computed values of THSA and experimental RT for epimers of triterpene and steroidal glycosides are presented in Figure 9. 
a)

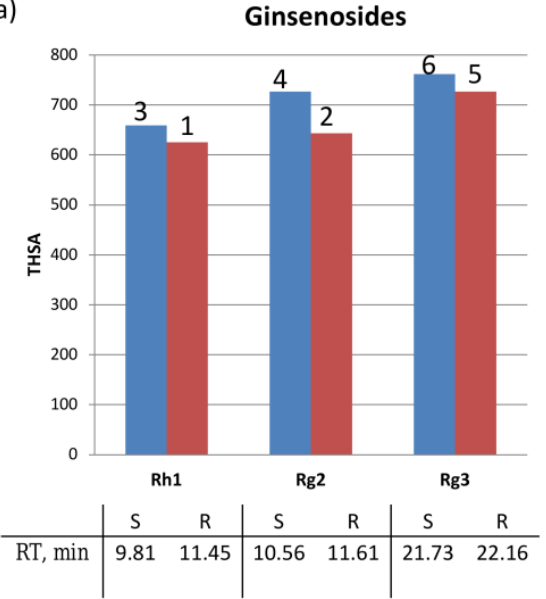

b)

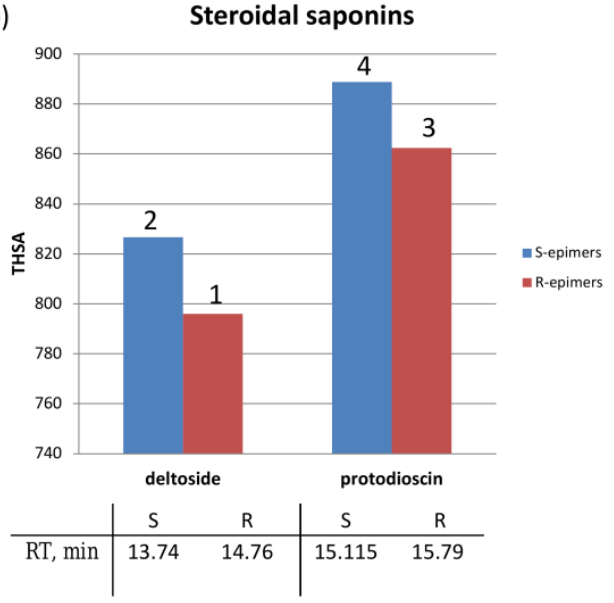

Figure 9. Chart of THSA with numbers indicating evaluated elution order and experimental RTs for S, R- (a) ginsenosides (b) and steroidal saponins.

S-epimers are systematically eluted earlier than R-epimers for both types of glycosides. THSA for both types of glycosides is truly correlated with retention, however, it has inverse dependence for both types of epimers. Uncommon results were obtained not only for THSA calculation. The rare behavior was observed for steroidal glycosides: an increase in temperature led to an increase in retention (Figure 10).

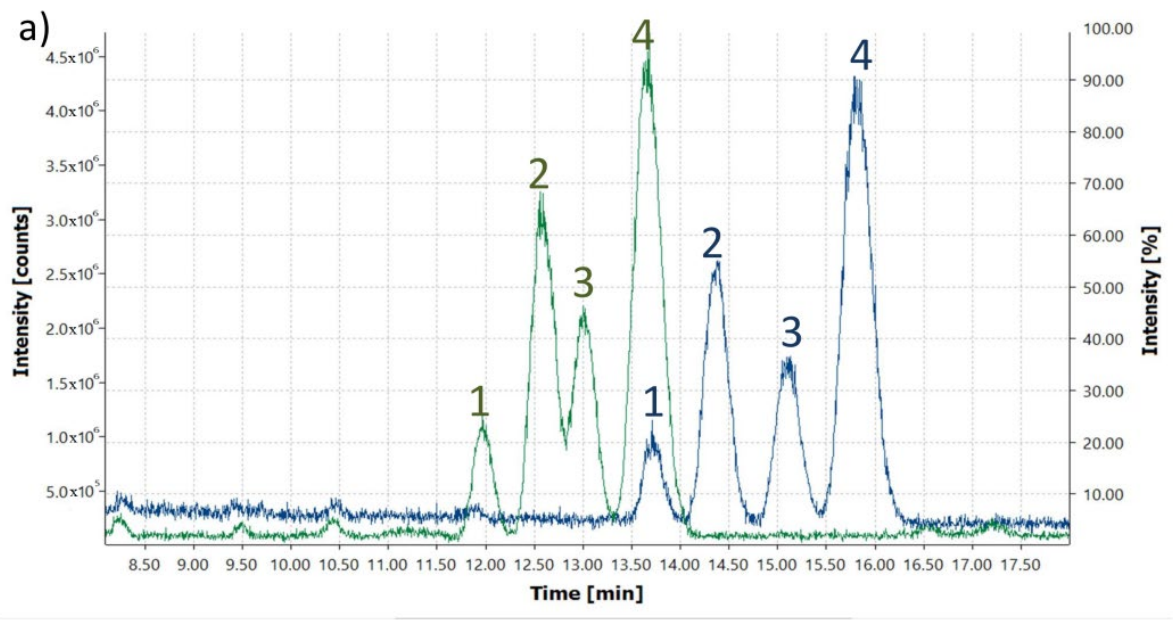

b)

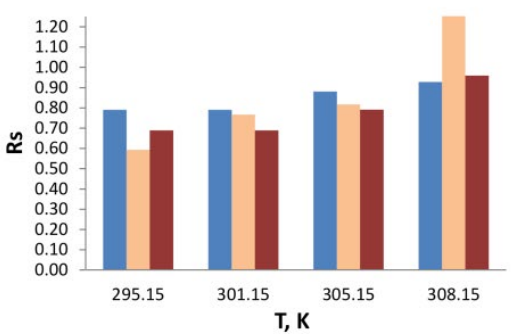

c)

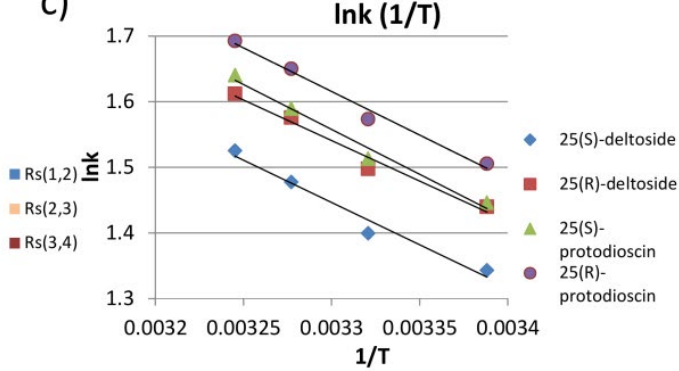

Figure 10. Chromatographic behavior of steroidal glycosides: (a) TIC obtained for $22{ }^{\circ} \mathrm{C}$ (green line) and $25^{\circ} \mathrm{C}$ (blue line) of steroidal saponins: 1-25(S)-deltoside, 2-24(R)-deltoside, 3-25(S)-protodioscin, and 4-25(R)-protodiosin. (b) Bar chart of Rs for three pairs and for four temperatures. (c) Van't Hoff plot for four temperatures.

Temperature effect was studied systematically: capacity factors were measured with four temperatures in the same chromatographic conditions. The resolution of epimers was better than for 25(S)-protodioscin and 25(R)-deltoside, which differ in the type of 
sugars. Moreover, an increase in temperature slightly influenced the resolution of deltoside epimers (1.17 times), the effect was less noticeable for protodioscin epimers (1.39 times) and significantly higher, almost up to the baseline, for 25(R)-deltoside and 25(S)-protodioscin (2.13 times). For every compound, Van't Hoff plots were built in order to study the retention mechanism. The natural logarithm of the capacity factor (lnk) is directly related to the energies of the chromatographic process with the approximate Equation (1):

$$
\ln \mathrm{k}=\frac{-\Delta \mathrm{H}^{\circ}}{\mathrm{RT}}+\frac{\Delta \mathrm{S}^{\circ}}{\mathrm{R}}+\ln \Phi
$$

This equation shows that if enthalpy $\left(\Delta \mathrm{H}^{\circ}\right)$, entropy $\left(\Delta \mathrm{S}^{\circ}\right)$, and phase ratio $(\Phi)$ are independent of temperature $(\mathrm{T})$, lnk versus $1 / \mathrm{T}$ is linear. These assumptions allow estimation of $\Delta \mathrm{H}^{\circ}$ and $\Delta \mathrm{G}^{\circ}$ of the adsorption process. The reduction of enthalpy regularly leads to the increase of $\mathrm{k}$ while reduce in entropy lowers the $\mathrm{k}$. Typical enthalpy interactions in reversed-phase systems are about -10 to $-15 \mathrm{~kJ} \mathrm{~mol}^{-1}$ for small molecules. In the case of steroidal glycosides, positive values of enthalpy were obtained. The positive enthalpy values are compensated with the entropy effect, thus leading to the linear decrease in Gibbs's energy with RT. The hydrophobic interaction concept is based on solvent relaxation (reduction of entropy) when the solute leaves the mobile phase and interacts with the stationary phase, which gives the main contribution to the hydrophobic interactions. These results indicate that the retention mechanism of the considered compounds cannot be simply described by the hydrophobic model. As can be seen in the Table 6, enthalpy calculated from Van't Hoff plots has the similar tendency with RT as THSA for epimers: enthalpy for $\mathrm{S}$ epimers is higher than for R. Reversed temperature effects could be caused either by secondary interactions [28], reduced ionization [28], or by reduced solubility in the mobile phase at higher temperatures [29].

Thereby, specific retention-temperature dependence can be explained by a combination of molecular structure peculiarities of saponins and stationary phase changes. Similar uncommon retention behavior of ginsenosides (triterpene saponins) was observed and studied in the article [30]. The authors concluded that the increase in retention time of both ginsenosides at elevated temperatures is affected by substitutes' arrangement. Thus, this suggestion about the role of saccharides can be applied to steroidal saponins. For such complex molecules, it is difficult to describe the retention mechanism using one simple parameter. TSHA correlates well with the release time of ginsenosides and steroids, but an inverse correlation with the value of this parameter is observed for epimers. Moreover, obtained correlations are representative only with RP with C18 stationary phase and acetonitrile as an organic modifier. Other RP stationary phases or organic solvents provide additional interaction in the separation mechanism. However, calculated properties could be useful in chromatographic analysis. Epimers can be separated with HPLC but cannot be distinguished with MS detection without standard compounds. Thus, the application of such theoretically obtained molecular properties could help to evaluate elution order for isomers. However, further investigations concerning the retention mechanism of ginsenosides with $\mathrm{C} 18$ bonded stationary phase are needed.

\section{Conclusions}

The thermodynamic characteristics of adsorption of a number of isomeric compounds were determined using molecular statistical calculations. It was found that the position of substituents plays a significant role in the adsorption processes. By-products in the chlorination of 4-phenylphenol were identified: 4-hydroxy-2,3'-and 3,2' -dichlorobiphenyls, 4-hydroxy-3,5,2' - and 2,3,6-trichlorobiphenyls. Performed GC-MS analysis and molecularstatistical calculations allowed to determine the main products of methoxybenzene dimerization: 3,4'- and 4,4'-dimethoxybiphenyls. The identified impurities were 3-methyl-, 4methyl-, and 5-methyl-2,2'-dimethoxybiphenyl. Elution order of isomeric chlorobiphenyls was predicted and compared with experimental data. 
Two simple calculated molecular properties were considered to describe the retention behavior of structural isomers and epimers of plant glycosides. Different partition coefficients used to characterize hydrophobicity were incapable of evaluating retention for complex molecules. THSA was found to be more representative in revealing the retention-isomerism relationship. Thermodynamic parameters using Van't Hoff plots were determined and used to describe the uncommon retention behavior of asymmetric molecules with a number of chiral centers epimers in RP-HPLC.

The symmetry of the considered molecule, as well as the symmetry of the stationary phase, should be taken into account during calculations due to the different sorption abilities of the asymmetric molecule in comparison with a symmetric molecule. However, in LC, taking into account the symmetry is much more complicated due to the complex analyte-stationary phase interactions and thus is an object of future studies.

Author Contributions: E.F., A.S., I.M. and A.B.: The authors contributed equally to this work. All authors have read and agreed to the published version of the manuscript.

Funding: This research was funded by the Ministry of Science and Higher Education of the Russian Federation grant number 075-15-2020-782.

Institutional Review Board Statement: Not applicable.

Data Availability Statement: The data presented in this study are available on request from the corresponding author.

Conflicts of Interest: The authors declare that they have no known competing financial interest or personal relationships that could have appeared to influence the work reported in this paper.

\section{References}

1. Slanina, Z. Theoretical Aspects of the Phenomenon of Isomerism in Chemistry; Pub. Mir.: Moscow, Russia, 1984.

2. Kalaivani, S. Isomerism in Organic Compounds; MJP Publishers: Chennai, India, 2013.

3. Becker, G. Introduction to the Electronic Theory of Organic Compounds; Pub. Mir.: Moscow, Russia, 1977.

4. Rafferty, J.L.; Zhang, L.; Siepmann, J.I.; Schure, M.R. Retention mechanism in reversed-phase liquid chromatography: A molecular perspective. Anal. Chem. 2007, 79, 6551-6558. [CrossRef]

5. Silva, C.; Ribeiro, C.; Maia, A.S.; Gonçalves, V.; Tiritan, M.E.; Afonso, C. Enantiomeric separation of tramadol and its metabolites: Method validation and application to environmental samples. Symmetry 2017, 9, 170. [CrossRef]

6. Clonazolam, B.; Rosetti, A.; Villani, C. Low Temperature Dynamic Chromatography for the Separation of the Interconverting Conformational Enantiomers of the Benzodiazepines Clonazolam, Flubromazolam, Diclazepam and Flurazepam. Symmetry 2021, 13, 1012.

7. Khandy, M.T.; Titova, M.V.; Konstantinova, S.V.; Kochkin, D.V.; Ivanov, I.M.; Nosov, A.M. Formation of protodioscin and deltoside isomers in suspension cultures of Nepal yam (Dioscorea deltoidea Wall.) cells. Appl. Biochem. Microbiol. 2016, 52, 657-662. [CrossRef]

8. Lee, S.M.; Kim, S.C.; Oh, J.; Kim, J.H.; Na, M. 20(R)-Ginsenoside Rf: A new ginsenoside from red ginseng extract. Phytochem. Lett. 2013, 6, 620-624. [CrossRef]

9. Chang, Y.H.; Ng, P.K.W. Effects of extrusion process variables on extractable ginsenosides in wheat-ginseng extrudates. J. Agric. Food Chem. 2009, 57, 2356-2362. [CrossRef]

10. Wang, X.; Zhao, T.; Gao, X.; Dan, M.; Zhou, M.; Jia, W. Simultaneous determination of 17 ginsenosides in rat urine by ultra performance liquid chromatography-mass spectrometry with solid-phase extraction. Anal. Chim. Acta 2007, 594, 265-273. [CrossRef]

11. Tawab, M.A.; Bahr, U.; Karas, M.; Wurglics, M.; Schubert-Zsilavecz, M. Degradation of ginsenosides in humans after oral administration. Drug Metab. Dispos. 2003, 31, 1065-1071. [CrossRef]

12. Gui, F.J.; Yang, X.W.; Li, L.Y.; Tian, J.M. Simultaneous enantiomer determination of 20 (R)- and 20 (S)-ginsenoside-Rg2 in rat plasma after intravenous administration using HPLC method. J. Chromatogr. B Anal. Technol. Biomed. Life Sci. 2007, 850, 1-6. [CrossRef]

13. Ma, L.Y.; Zhang, Y.B.; Zhou, Q.L.; Yang, Y.F.; Yang, X.W.; McPhee, D.J. Simultaneous determination of eight ginsenosides in rat plasma by liquid chromatography-electrospray ionization tandem mass spectrometry: Application to their pharmacokinetics. Molecules 2015, 20, 21597-21608. [CrossRef]

14. Kouskoura, M.G.; Mitani, C.V.; Markopoulou, C.K. A complementary study of mechanisms and behaviors in chromatography via modeling. J. AOAC Int. 2015, 98, 1462-1470. [CrossRef] [PubMed]

15. Kiselev, V.; Grumadas, J.; Lithuanian, S.S.R. Statistical Molecular Calculation of Thermodynamic Parameters. J. Chem. Soc. Faraday Trans. 1 Phys. Chem. Condens. Phases 1977, 75, 1281-1287. 
16. Matyushin, D.D.; Ukleina, A.N.; Buryak, A.K. A Comparative Study of Adsorption of Isomeric Molecules on Carbon Sorbents from a Gas and a Liquid. Prot. Met. Phys. Chem. Surfaces 2020, 56, 38-43. [CrossRef]

17. Terent'ev, A.V.; Varfolomeeva, V.V.; Buryak, A.K. Calculation of Henry Constants for the Adsorption of Isomeric Phenylenediamines on Graphitized Thermal Carbon Black. Russ. J. Phys. Chem. A 2009, 83, 2331-2335. [CrossRef]

18. Moshkarina, N.A.; Dianova, I.A.; Lebedev, A.T.; Buryak, A.K.; Petrosyan, V.S. The Aqueous Chlorination of the Structural Fragments of Humic Matter. In Proceedings of the 5th International Conference on Water Pollution: Modeling, Measuring and Prediction. Water Pollution 99, Lemnos, Greece, 24-26 May 1999; WIT Press: Ashurst, UK, 1999; pp. 515-524.

19. Panzella, L.; Manini, P.; Napolitano, A.; D’Ischia, M. Free radical oxidation of (E)-retinoic acid by the Fenton reagent: Competing epoxidation and oxidative breakdown pathways and novel products of 5,6-epoxyretinoic acid transformation. Chem. Res. Toxicol. 2004, 17, 1716-1724. [CrossRef]

20. Carfagna, C.; Amendola, E.; Giamberini, M.; D'Amore, A.; Priola, A.; Malucelli, G. The effect of prepolymer composition of amino-hardened liquid crystalline epoxy resins on physical properties of cured thermoset. Macromol. Symp. 1999, 148, 197-209. [CrossRef]

21. Naumov, V.A.; Kataeva, O.N. Molecular Structure of Organic Compounds of Oxygen and Sulfur in the Gas Phase; Science: Moscow, Russia, 1990.

22. Grumadas, J.; Poshkus, P.; Kiselev, V. Determination of Molecular Structure Parameters on the Basis of Gas-adsorption Chromatography Data. J. Chem. Soc. Faraday Trans. 2 Mol. Chem. Phys. 1982, 78, 2013-2023.

23. Afarinkia, K.; Aitken, R.; Armstrong, A. Comprehensive Organic Functional Group Transformations; Pergamon Press: Oxford, UK, 1995; Volume 1, pp. 71-103.

24. Parent, R.A. Methylene chloride. Encycl. Toxicol. 1995, 15, 92-95.

25. Kalashnikova, E.V.; Kiselev, A.V.; Shcherbakova, K.D.; Vasileva, S.D. Retention of diphenyls, terphenyls, phenylalkanes and fluorene on graphitized thermal carbon black. Chromatographia 1981, 14, 510-514. [CrossRef]

26. Sovocool, G.W.; Mitchum, R.K.; Donnelly, J.R. Use of the 'ortho effect' for chlorinated biphenyl and brominated biphenyl isomer identification. Biomed. Environ. Mass Spectrom. 1987, 14, 579-582. [CrossRef]

27. Stanton, D.T.; Jurs, P.C. Development and Use of Charged Partial Surface Area Structural Descriptors in Computer-Assisted Quantitative Structure-Property Relationship Studies. Anal. Chem. 1990, 62, 2323-2329. [CrossRef]

28. McCalley, D.V. Effect of temperature and flow-rate on analysis of basic compounds in high-performance liquid chromatography using a reversed-phase column. J. Chromatogr. A 2000, 902, 311-321. [CrossRef]

29. Lochmüller, C.H.; Moebus, M.A.; Liu, Q.; Jiang, C.; Elomaa, M. Temperature effect on retention and separation of poly(ethylene glycol)s in reversed-phase liquid chromatography. J. Chromatogr. Sci. 1996, 34, 69-76. [CrossRef]

30. Soliev, A.; Quiming, N.S.; Ohta, H.; Saito, Y.; Jinno, K. Separation of ginsenosides at elevated temperature by ultra high pressure liquid chromatography. J. Liq. Chromatogr. Relat. Technol. 2007, 30, 2835-2849. [CrossRef] 